\title{
Stratégies d'apprentissage d'une langue étrangère et traitement pédagogique : aperçu d'une méthodologie de recherche
}

Janet Atlan

\section{OpenEdition}

\section{Journals}

Édition électronique

URL : http://journals.openedition.org/asp/3985

DOI : 10.4000/asp.3985

ISSN : 2108-6354

Éditeur

Groupe d'étude et de recherche en anglais de spécialité

\section{Édition imprimée}

Date de publication : 1 décembre 1995

Pagination : 453-471

ISSN : 1246-8185

\section{Référence électronique}

Janet Atlan, «Stratégies d'apprentissage d'une langue étrangère et traitement pédagogique : aperçu d'une méthodologie de recherche », ASp [En ligne], 7-10 | 1995, mis en ligne le 19 novembre 2013, consulté le 01 mai 2019. URL : http://journals.openedition.org/asp/3985 ; DOI : 10.4000/asp.3985

Ce document a été généré automatiquement le 1 mai 2019.

Tous droits réservés 


\title{
Stratégies d'apprentissage d'une langue étrangère et traitement pédagogique : aperçu d'une méthodologie de recherche
}

\author{
Janet Atlan
}

1 En matière d'enseignement et d'apprentissage, étudier l'interaction entre aptitude et traitement pédagogique (Aptitude Treatment Interaction, ATI), c'est étudier les effets sur l'apprentissage de la mise en relation de certaines caractéristiques de l'apprenant (les différences individuelles) avec des outils pédagogiques. Beaucoup de recherches sur l'interaction aptitude/traitement pédagogique et l'apprentissage ont été faites par les psychologues cognitifs depuis les années 1960 (voir en particulier Salomon, 1974 ; Clark 1983 ; Olson 1972 et Cronbach \& Snow 1977), mais peu de chercheurs se sont penchés sur l'ATI appliquée à l'apprentissage d'une langue étrangère/seconde (voir néanmoins Skehan 1989, pour un aperçu des travaux dans ce domaine).

2 Cependant les recherches nombreuses qui ont porté, depuis le début des années 1970, sur l'identification et la classification des différences individuelles en apprentissage des langues nous donnent maintenant une base solide pour des travaux dans ce domaine. L'utilisation de plus en plus fréquente des nouvelles technologies pour l'enseignement des langues étrangères a déjà donné lieu à beaucoup d'études sur l'impact de ces technologies sur l'apprentissage. Il est donc intéressant d'utiliser ce que nous savons afin d'étudier l'interaction entre les deux phénomènes.

3 L'objectif est ici de décrire une méthodologie de recherche que nous développons pour étudier les liens qui pourraient exister entre les différences individuelles et le support média utilisé lors d'une tâche d'apprentissage de langue étrangère. Lors de cette étude, les stratégies d'apprentissage seront considérées comme un cas particulier des différences individuelles. Le travail décrit en est encore au stade du développement d'outils pour évaluer les stratégies utilisées en présence de différents supports médias, et il faut prévoir d'appliquer ces outils à un public plus nombreux que le public testé et à 
d'autres tâches que celles décrites. Cependant nous pensons qu'il est intéressant d'étudier l'interaction entre les stratégies d'apprentissage et le média utilisé, car si nous savons comment les apprenants travaillent, nous pourrons mieux construire les tâches d'apprentissage en cours de langue.

La présentation sera en trois parties. D'abord nous donnerons un aperçu des bases théoriques en présentant le modèle d'apprentissage d'une langue étrangère utilisé, la place des différences individuelles dans cet apprentissage et l'utilisation des technologies éducatives dans l'enseignement d'une langue étrangère. Ensuite nous décrirons la méthode de travail en présentant le protocole de recherche et nous présenterons rapidement quelques résultats obtenus.

\section{Le modèle du « bon apprenant en langues » comme cadre de travail}

Parmi les divers modèles d'apprentissage d'une langue étrangère qui ont été élaborés, le Monitor Model de Dulay, Burt et Krashen (1982) et le Good Language Learner Model de Naiman et al. (1978) sont parmi les plus connus. Le modèle du moniteur est un modèle classique dans la mesure où il part de prémisses et produit des prédictions. En revanche, le terme « modèle » pour la description du « bon apprenant en langues » est trompeur car ce modèle est essentiellement une liste de variables dans l'apprentissage d'une langue et ne se réfère pas explicitement à des théories de l'apprentissage d'une langue.

6 Cependant, en parlant de ces deux modèles comme base de recherche sur les différences individuelles, Skehan (1989) note que le modèle du bon apprenant en langues a trois avantages sur le modèle du moniteur. D'abord il met en évidence les différents paramètres d'un apprentissage réussi. Ensuite il se prête à la quantification des différents paramètres car il permet de montrer dans quelle mesure une variable donnée a un effet sur une autre variable. Enfin il permet de conceptualiser les effets de l'interaction. Comme il y a une liste de paramètres potentiels, la recherche des interactions est plus aisée.

7 Skehan (1989: 3) fait remarquer, au contraire, qu'avec le modèle du moniteur

... there is only room for universal processes and lack of individual differences. The assumption being made that given comparable input, all learners will process the data in the same way and at the same speed.

8 De plus, le modèle du bon apprenant en langues, avec sa catégorisation de variables pertinentes, se prête à une approche empirique de la recherche. Cette approche consiste en l'identification d'un domaine bien défini pour la recherche et la collecte de données dans ce domaine. Ces données peuvent être utilisées ensuite pour élaborer une théorie. En effet, l'étude des différences individuelles peut être conçue plus facilement à l'intérieur d'une telle approche et le modèle du bon apprenant en langues semble donc être approprié comme cadre de recherche sur l'interaction entre les différences individuelles et le traitement pédagogique dans l'apprentissage de langues étrangères (Skehan 1989).

9 La figure 1 est un schéma des variables intervenant en apprentissage des langues étrangères fondé sur le modèle du bon apprenant de Naiman et élaboré par Skehan (1989). Nous en avons revu la mise en page afin de le rendre plus compréhensible. 
Figure 1: Le modèle du « bon apprenant en langues »

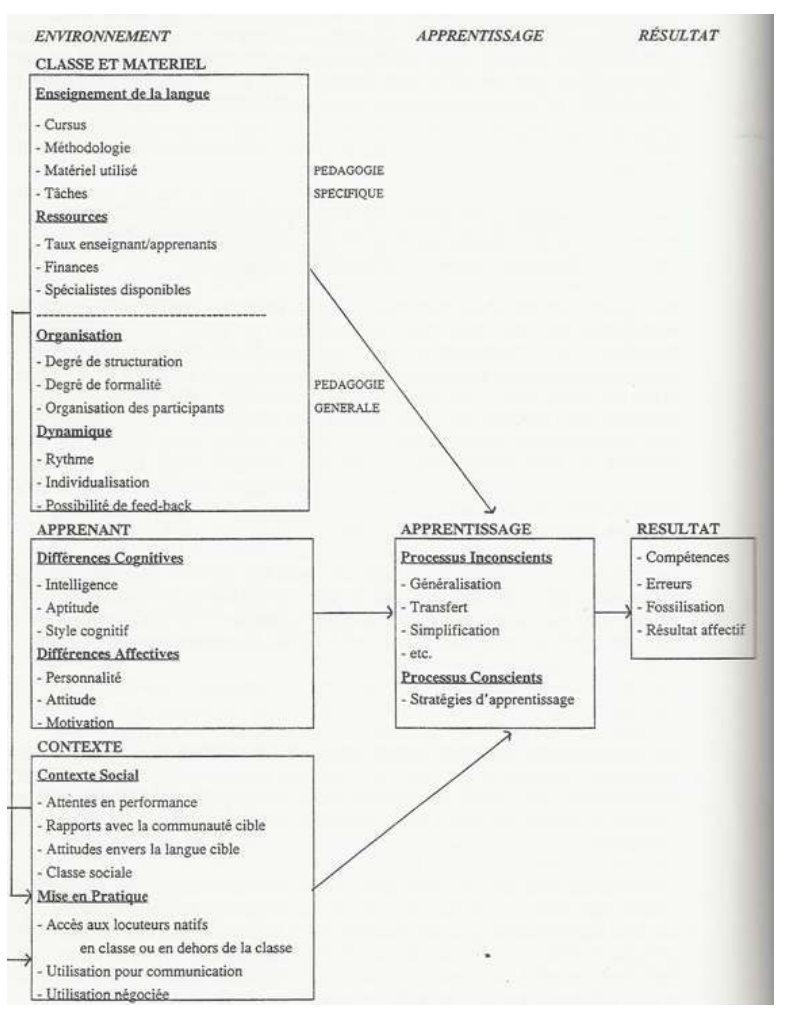

Adapté de Naiman et al. (1978) et Skehan (1989)

Le modèle se décompose en cinq groupes de variables en apprentissage des langues avec trois groupes de variables indépendantes («classe et matériel», «apprenant» et « contexte ») et deux groupes de variables dépendantes (" apprentissage » et « résultat »). Chaque groupe est ensuite décomposé en plusieurs paramètres. Le choix et l'appellation des différents éléments dans chaque groupe peuvent être discutés mais le schéma de base reste inchangé.

11 Les flèches indiquent les influences que les différentes classes de variables ont les unes sur les autres. Au début de l'apprentissage il y a un état initial décrit par l'environnement et un résultat est atteint après passage par un processus d'apprentissage. À l'intérieur de l'environnement, on peut voir aussi que l'élément «classe et matériels » a une influence sur le « contexte » et que le « contexte social » joue un rôle dans la mise en pratique de la langue.

\section{Les différences individuelles en apprentissage des langues étrangères}

Dans cette partie, nous donnerons un cadre conceptuel aux différences individuelles en nous appuyant principalement sur un modèle cognitif de l'apprentissage. La recherche sur les différences individuelles s'attache plus aux différences entre les apprenants qu'aux similitudes. Ce contraste entre deux types de recherche est courant en psychologie mais pas en apprentissage de langues (Skehan 1989). 
L'essentiel de la recherche faite sur les différences individuelles en apprentissage de langues est centré sur l'effet de ces différences sur le résultat de l'apprentissage plutôt que sur le processus d'apprentissage lui-même. Avec l'aide du modèle du bon apprenant, nous proposons d'inclure les stratégies d'apprentissage dans le processus d'apprentissage et d'examiner l'influence du traitement pédagogique sur leur utilisation par l'apprenant.

Pour ce faire, nous séparerons les différences individuelles en deux catégories. Une catégorie de variables décrit les aspects cognitifs, affectifs et socioculturels de l'individu au début de l'apprentissage. Ce sont des variables qui sont en grande partie inconscientes et incontrôlables par l'individu. Elles sont susceptibles de changer avec le temps mais nous supposons qu'elles sont stables au moment d'exécuter une tâche donnée. Par contre, les stratégies sont des techniques que l'individu peut mobiliser pour faciliter et faire avancer l'apprentissage.

Les stratégies d'apprentissage peuvent être définies comme des connaissances métacognitives qui permettent de savoir quand et comment utiliser des processus et des données déjà à la disposition de l'apprenant. Le terme fait référence à la fois aux comportements réels des apprenants, à ce que les apprenants savent sur l'utilisation des stratégies et à ce que les apprenants savent sur l'apprentissage d'une langue (Wenden 1987). Brown et al. (1983) ont identifié trois facteurs qui jouent dans le choix de stratégies: la tâche à accomplir, les caractéristiques de l'apprenant et la nature du matériel pédagogique.

Wenden (1987) caractérise les stratégies suivant six critères. Ce sont des actions ou techniques spécifiques à la différence de « réflexion » ou « prise des risques » qui entrent dans le style cognitif. Certaines peuvent être observées par le chercheur, d'autres non. Elles sont orientées vers un problème et facilitent l'acquisition, le stockage, la récupération ou l'utilisation des données. Elles peuvent contribuer directement ou indirectement à l'apprentissage. Elles peuvent être utilisées consciemment mais peuvent devenir automatiques et elles restent potentiellement conscientes. Elles sont négociables et peuvent être modifiées, rejetées et/ou apprises.

Selon Wenden (1987: 6),

research on learner strategies in the domain of second language learning may be viewed as part of a general area of research on mental processes and structure that constitute the field of cognitive science.

8 Cependant la recherche faite dans les années 1970 et 80 sur l'utilisation des stratégies dans l'apprentissage d'une langue a surtout donné lieu à des systèmes de classement plutôt qu'à l'étude de leur place dans le processus d'apprentissage. Trois classements de stratégies d'apprentissage émergent.

La typologie élaborée par Rubin en 1981 était une des premières tentatives de classement de stratégies. Elle a distingué les stratégies d'apprentissage qui contribuent directement à l'apprentissage et au développement du système langagier des stratégies dites de communication et sociales qui sont indirectes. De plus, elle a classé les stratégies d'apprentissage en stratégies métacognitives (connaissances sur et régulation des processus cognitifs) et stratégies cognitives (opérations qui exigent l'analyse directe, la synthèse ou la transformation de la matière linguistique). O'Malley et Chamot (1987) ont été les premiers à établir une liste précise des stratégies cognitives et métacognitives en y ajoutant une troisième classe de stratégies qui prend en compte les actions sociales et affectives de l'apprenant. En 1990, Oxford a élaboré un classement qui diffère de celui 
d'O'Malley et Chamot en ce que la dichotomie principale se trouve entre stratégies directes et indirectes. Les stratégies directes sont divisées en stratégies "de rappel ", «cognitive» ou «de compensation». Les stratégies indirectes qui n'opèrent pas directement sur la langue correspondent aux stratégies métacognitives et socio-affectives d'o'Malley et Chamot.

Puisque les différences individuelles sont difficiles à observer, la question de savoir comment les données de la recherche sont recueillies est importante. Les variables cognitives ont été largement étudiées et il existe de nombreux outils qui permettent de les définir pour un individu donné. De même, les variables affectives et socioculturelles ont fait l'objet de nombreux questionnaires auprès des apprenants.

En ce qui concerne les stratégies d'apprentissage, Oxford et Crookall (1989) ont identifié six méthodes pour recueillir des données. Ce sont des listes faites par le chercheur et fondées sur l'observation et l'intuition, les entretiens et autres procédures de réflexion à haute voix, la prise de notes par les apprenants, la tenue d'un journal par les apprenants, les questionnaires et sondages administrés par le chercheur et les données recueillies lors d'un entraînement à l'utilisation des stratégies d'apprentissage. Les méthodes les mieux adaptées à l'étude d'un grand nombre de sujets sont les questionnaires et sondages avec des entretiens pour approfondir les réponses de certaines personnes.

En général, le recueil de données sur les stratégies d'apprentissage implique qu'on demande à l'apprenant ce qu'il fait pendant qu'il exécute une tâche. Cohen (1987) identifie trois types de compte rendu oral. Lors d'un retour sur soi (self-report), les apprenants émettent des commentaires d'ordre général sur leur comportement. L'autoobservation (self-observation) est un compte rendu sur un comportement spécifique lors d'une tâche donnée. Ce compte rendu peut être introspectif (immédiatement à la fin de la tâche) ou rétrospectif (plus tard, après la fin de la tâche). L'auto-révélation (self-revelation) est un compte rendu des processus mentaux qui est exécuté en même temps que la tâche.

En ce qui concerne l'importance des différences individuelles au cours de l'apprentissage d'une langue étrangère, Skehan (1989) soutient que les résultats de la recherche sur les différences individuelles ont été largement ignorés par la recherche sur l'acquisition des langues étrangères. Même s'ils appartiennent à une tradition de recherche empirique, ils devraient être un élément important dans une approche hiérarchique. Ils nous permettent d'apercevoir l'importance du problème que des études futures auront à traiter.

\section{La technologie éducative et l'apprentissage des langues}

Nous passons maintenant à une description de la place de la technologie éducative dans l'apprentissage d'une langue étrangère. Pour ce faire, il nous faut d'abord définir l'expression «technologie éducative». Ensuite, nous proposerons un schéma de communication qui nous permet d'intégrer la technologie dans le processus de l'apprentissage et nous donnerons quelques caractéristiques des différents supports médias utilisés pour l'enseignement.

Galbraith (1967) a défini la technologie comme étant l'application systématique des connaissances ordonnées et/ou scientifiques à des tâches pratiques. L'enseignement est la mise en scène de l'information et de l'environnement (le lieu de l'apprentissage, les 
méthodes, les médias et l'équipement) de façon à faciliter l'apprentissage. L'apprentissage est l'élaboration de nouvelles connaissances, compétences et attitudes lors de l'interaction de l'individu avec son environnement. Une technologie éducative est donc un arrangement systématique des événements d'apprentissage et/ou d'enseignement destiné à mettre en pratique les connaissances sur l'apprentissage de façon prévisible et efficace afin d'atteindre des objectifs d'apprentissage définis (Heinich et al. 1993). Un schéma de la communication qui nous permet d'analyser les situations d'enseignement est celui de Shannon et Weaver (1949). La figure 2 montre comment ce schéma peut être mis en parallèle avec l'apprentissage des langues.

Figure 2: Modèle de la communication Shannon-Weaver et son intégration dans l'environnement pédagogique

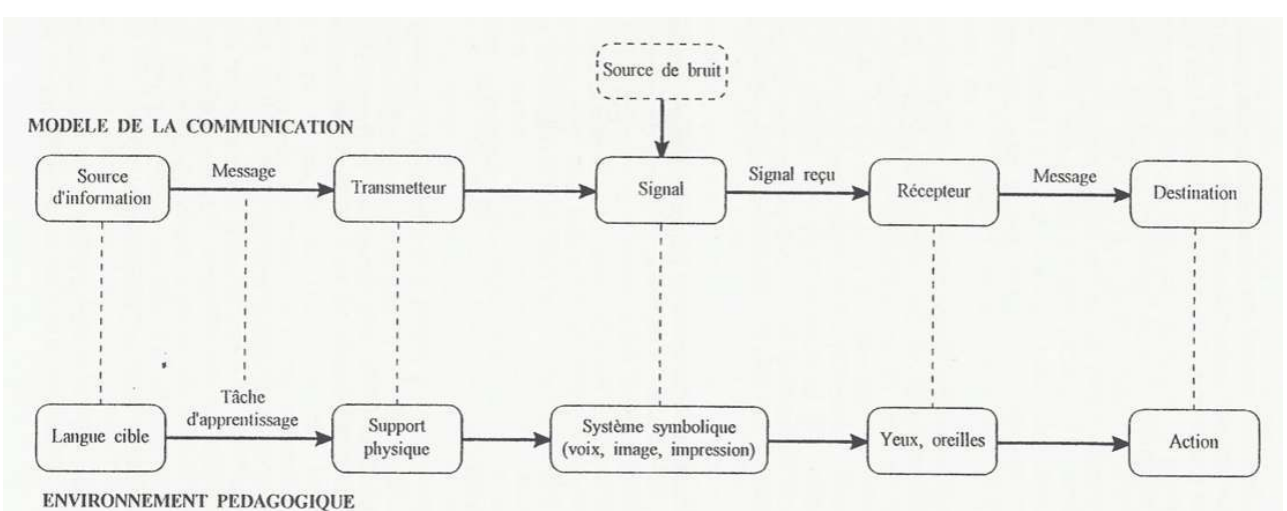

Si nous postulons que la technologie éducative se compose à la fois de la tâche d'apprentissage, du support physique par lequel le message est transmis et du système symbolique, nous pouvons comprendre comment il s'intègre dans le schéma de communication.

Dans le schéma de la communication, le support est réduit au rôle de transmetteur. En fait, le terme est généralement utilisé pour parler d'un canal de communication (film, télévision, diagramme, document imprimé, ordinateur, voix de l'enseignant, etc.) qui porte l'information entre la source et le récepteur. Dans ce cas, il porte le message à la fois comme transmetteur et signal. Afin d'intégrer le support dans l'enseignement, il faut incorporer la notion de méthode. Une méthode est une procédure d'enseignement qui a été sélectionnée pour aider les apprenants à atteindre les objectifs voulus ou à assimiler le contenu d'un message (Heinich et al. 1993). Elle peut comporter des éléments tels que la présentation, la démonstration, l'exercice de répétition, la simulation, l'étude de cas, etc. Heinich et al. illustrent le rôle du support de la façon suivante (voir figure 3). 
Figure 3

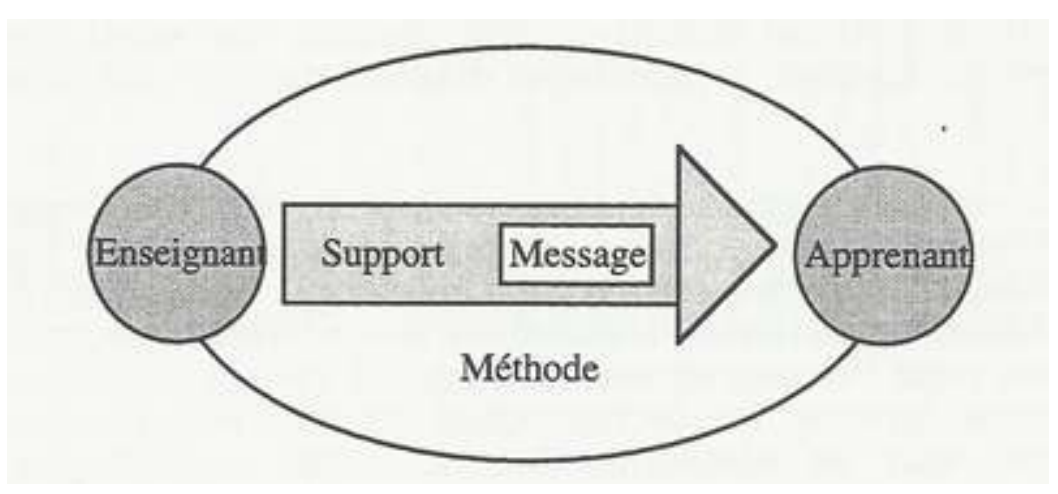

Le message est porté par le support et la méthode est la situation d'apprentissage. Bien que Clark (1983) soutienne que les différences dans les résultats d'apprentissage [quand on utilise la technologie éducative] sont le fait de la méthode utilisée, et non du support, Kozma (1991) nous fait remarquer que ceci crée une rupture inutile, car le support et la méthode ont des rôles complémentaires dans la conception de l'enseignement. Le support peut permettre et contraindre la méthode à la fois, tandis que la méthode peut tirer parti du support tout en mobilisant ses atouts.

Cette discussion montre que la technologie est un processus et non seulement une collection de produits. On pourrait faire une distinction entre technologie dure et technologie molle: la technologie dure serait le support, la technologie molle serait la méthode ou le cadre dans lequel on organise l'enseignement.

Un média utilisé pour l'enseignement comporte trois attributs pertinents pour la cognition: une technologie, un système symbolique et des capacités de traitement (Kozma 1991). Les aspects mécaniques et électroniques qui déterminent sa fonction constituent la technologie d'un média. Les effets cognitifs de la technologie, s'ils existent, sont minimes. En fait, la technologie d'un média a pour effet surtout de limiter les deux autres attributs.

2 Les systèmes symboliques sont des modes de présentation (mots, illustrations, etc.) régis à l'intérieur de chaque système par une syntaxe et utilisés de façon spécifique par rapport à des champs de référence. Un média peut être décrit et distingué d'un autre média par sa capacité à employer un système symbolique donné. Les systèmes symboliques ont une influence importante sur la manière dont un apprenant représente et traite l'information venant d'un support média.

Un média est aussi décrit et caractérisé par ses capacités spécifiques qui servent à traiter son système symbolique. Le fait d'inclure des attributs concernant le traitement dans la définition d'un média sert à créer une distinction entre deux médias qui ont le même système symbolique (par exemple, vidéodisque et émission télévisée). Les capacités de traitement d'un média peuvent compléter celles d'un apprenant, et chaque média a pour résultat des effets cognitifs spécifiques. Le tableau 1 montre les attributs cognitifs des différents médias. 
Tableau 1 : Les attributs cognitifs des médias

\begin{tabular}{|c|c|c|c|c|}
\hline & TECHNOLOGIE & SYSTĖME SYMBOLIQUE & CAPACITÉS DE TRAITEMENT & EFFETS COGNITIFS \\
\hline LIVRE & $\begin{array}{l}\text { Page imprimée } \\
\text { Portable }\end{array}$ & $\begin{array}{l}\text { Visuel linguistique (texte, symboles } \\
\text { orthographiques) } \\
\text { Visuel iconique (images) }\end{array}$ & $\begin{array}{l}\text { Stable } \\
\text { Image fixe } \\
\text { Traitement linéaire } \\
\text { Vitesse individuelle } \\
\text { Retour en arrière possible }\end{array}$ & $\begin{array}{l}\text { Construction d'une base textuelle et } \\
\text { transposition sur un modèle mental de la } \\
\text { situation }\end{array}$ \\
\hline VIDEO/T.V. & \begin{tabular}{|l|} 
Ecran \\
Haut-parleur \\
Semi-portable
\end{tabular} & \begin{tabular}{|l|} 
Visuel iconique \\
Audio iconique (musique) \\
Audio linguistique \\
(Visuel linguistique)
\end{tabular} & $\begin{array}{l}\text { Transitoire } \\
\text { Mouvement } \\
\text { Traitement linéaire } \\
\text { Chronologique } \\
\text { Pas de retour en arrière } \\
\end{array}$ & $\begin{array}{l}\text { Traitement simultané de l'auditif et du } \\
\text { visuel }\end{array}$ \\
\hline AUDIO/RADIO & \begin{tabular}{|l} 
Bande magnétique \\
Bande digitale \\
Portable \\
Haut-parleur \\
\end{tabular} & $\begin{array}{l}\text { Audio linguistique } \\
\text { Audio iconique }\end{array}$ & \begin{tabular}{|l|} 
Transitoire \\
Traitement linéaire \\
Chronologique \\
Pas de retour en arrière \\
\end{tabular} & $\begin{array}{l}\text { Traitement de l'auditif et construction de } \\
\text { modèles mentaux }\end{array}$ \\
\hline ORDINATEUR & $\begin{array}{l}\text { Mémoire } \\
\text { Écran } \\
\text { Semi-portable }\end{array}$ & \begin{tabular}{|l} 
Visuel linguistique \\
Visuel iconique
\end{tabular} & $\begin{array}{l}\text { Stable } \\
\text { Image fixe } \\
\text { Traitement non-linéaire } \\
\text { Retour en arrière possible } \\
\text { Découpage et analyse de l'information } \\
\text { Transformation de l'information d'un système } \\
\text { symbolique à un autre } \\
\end{array}$ & $\begin{array}{l}\text { Construction et définition de modèles } \\
\text { mentaux }\end{array}$ \\
\hline $\begin{array}{l}\text { MULTIMÉDIA: } \\
\text { VIDEO : } \\
\text { INTERACTIVE }\end{array}$ & \begin{tabular}{|l|} 
Mémoire \\
Écran \\
Haut-parleur \\
Non-portable
\end{tabular} & \begin{tabular}{|l|} 
Visuel iconique \\
Audio iconique \\
Visuel linguistique \\
Audio linguistique
\end{tabular} & $\begin{array}{l}\text { Stable et/ou transitoire } \\
\text { Présentation simultanée du mouvement et d'une } \\
\text { image fixe } \\
\text { Traitement non-linéaire } \\
\text { Retour en arrière possible } \\
\text { Représentation visuelle et spatial dynamique } \\
\text { Création de réalité virtuelle }\end{array}$ & $\begin{array}{l}\text { Résolution de problèmes sémantiquement } \\
\text { plus riches }\end{array}$ \\
\hline $\begin{array}{l}\text { MULTIMÉDIA: } \\
\text { HYPERTEXTE }\end{array}$ & \begin{tabular}{|l} 
Mémoire \\
Écran \\
Semi-portable
\end{tabular} & \begin{tabular}{|l|} 
Visuel iconique \\
Visuel linguistique
\end{tabular} & $\begin{array}{l}\text { Stable } \\
\text { Présentation simultanée du texte et de l'image } \\
\text { Retour en arrière possible } \\
\text { Traitement non-linéaire } \\
\text { Placer des systèmes symboliques dans autres } \\
\text { contextes }\end{array}$ & $\begin{array}{l}\text { Transfert de l'information complexe à de } \\
\text { nouvelles situations }\end{array}$ \\
\hline
\end{tabular}
doit partir d'une expérience directe, passer par une représentation iconique de cette expérience et aboutir à une représentation symbolique. L'utilisation des nouvelles technologies éducatives pour l'enseignement correspond bien à ce continuum, étant donné qu'avec les technologies les plus récentes on peut représenter tous les éléments. Ces nouvelles technologies sont particulièrement pertinentes pour l'apprentissage d'une langue où les compétences communicatives nécessitent l'expérience directe et le système linguistique représente le composant symbolique.

De plus, Snow et Salomon ont fait remarquer $(1968: 342)$ que

instructional technology must be conceptualized as some combination of learning theory and individual differences because individual differences must be ranked among the most important independent variables in the study of complex learning.

Finalement, Garrett (1991) note qu'il est devenu courant de penser à la technologie comme un soutien à l'apprentissage plutôt que comme une aide à l'enseignement.

Nous postulons que les études existantes ne tiennent pas suffisamment compte des différences individuelles dans le traitement cognitif et dans la mise en œuvre des stratégies, car ces études sont conçues pour mesurer le résultat de l'apprentissage et non l'apprentissage lui-même. En effet, la synthèse de la recherche de Kozma (1991: 179) suggère que les capacités d'un support média donné, combinées avec des méthodes qui tirent parti de ces capacités, influent sur la façon dont un apprenant perçoit et traite l'information. Cette conception de l'apprentissage au moyen des technologies éducatives comme une interaction continue entre l'individu et l'environnement, entre l'apprenant et l'information médiatisée, est ce que Salomon (1994) et d'autres cogniciens commencent à étudier sous l'appellation distributed cognitions. Ce phénomène est à l'origine de l'expérience dont le compte rendu suit. 


\section{Le protocole de travail}

L'objet de cette expérience était de comparer les stratégies d'apprentissage de l'anglais utilisées habituellement par les sujets (telles que rapportées par eux) aux stratégies utilisées dans un contexte donné (aussi rapportées par les participants).

Pour ce faire, nous avons créé des tâches d'apprentissage dont le principe de base était toujours le même (compréhension et manipulation de vocabulaire dans un contexte technique), mais dont la présentation variait. L'information à traiter était présentée au moyen d'un parmi quatre supports possibles: cassette audio, cassette vidéo, papier ou écran d'ordinateur.

Chaque tâche comprenait un exercice lacunaire à compléter, des questions sur le contenu auxquelles il fallait répondre et quelques mots à traduire. De plus, quelques indications grammaticales ont été fournies avec le texte papier et le texte sur ordinateur pour aiguiller les participants, car les deux exercices nous semblaient un peu difficiles à faire sans aide.

Les textes utilisés pour les tâches étaient les suivants :

- Cassette audio en laboratoire de langues : Data Transmission extrait de English for Information Technology.

- Cassette vidéo travaillée individuellement : extrait du film How Does a Computer Work?

- Support papier: Fish that Can't See extrait de Minimum Competence in Scientific English.

- Travail sur écran ordinateur: The Growth of Salmon Farming extrait du logiciel Enigma 1 (Minimum Competence in Scientific English).

2 Les participants étaient des étudiants en première année d'études d'informatique en IUT. Leur niveau en anglais était très variable. Dans un premier temps, chaque participant a répondu à deux questionnaires administrés lors du cours d'anglais hebdomadaire par l'enseignant habituel de chaque groupe d'étudiants.

3 Le premier questionnaire (Q1) était le Strategy Inventory for Language Learning (élaboré par Oxford, 1990). Ce questionnaire se présente sous forme d'affirmations pour lesquelles le sujet doit noter son accord ou désaccord sur une échelle de Likert à cinq niveaux. Les affirmations peuvent être groupées pour correspondre aux six catégories de stratégies d'apprentissage définies par Oxford : stratégies de rappel, stratégies cognitives, stratégies de compensation, stratégies métacognitives, stratégies affectives et stratégies sociales. Les réponses à ce questionnaire donnent la fréquence d'utilisation des différents types de stratégies pour chaque participant.

Le deuxième questionnaire $(\mathrm{Q} 2)$, d'ordre général, portait sur le niveau en anglais et les opinions concernant l'apprentissage de langues et était destiné à fournir des renseignements dont nous aurions éventuellement besoin lors de l'analyse des résultats.

Lors d'une deuxième séance (une ou deux semaines plus tard), les participants ont été répartis au hasard dans des salles différentes pour exécuter une des quatre tâches prévues. Chacun a donc travaillé soit avec une cassette audio, soit avec une cassette vidéo, soit avec un ordinateur, soit avec un texte papier. Les sujets avaient la possibilité de communiquer entre eux ou avec l'enseignant et ils avaient des dictionnaires unilingues et bilingues à leur disposition. 
édiatement après l'exercice, chaque participant a répondu à un questionnaire (Q3) élaboré par nous-mêmes et destiné à faire émerger les techniques utilisées lors de l'exercice. Les réponses à ce questionnaire sont sous la forme oui/non ou jamais/parfois/ souvent ou réponse libre. Chaque question posée se rapporte à une stratégie d'apprentissage dans la classification d'Oxford. Il est donc possible encore une fois de bâtir le profil des stratégies utilisées par chaque participant.

\section{Analyse des résultats}

Après avoir terminé les séances de travail avec tous les participants, nous avions 94 dossiers exploitables (un jeu complet de trois questionnaires plus les réponses à l'exercice). Ces dossiers étaient repartis de la façon suivante : 26 pour la cassette audio, 21 pour la cassette vidéo, 22 pour le support papier et 25 pour l'écran ordinateur.

Nous avons procédé au codage des réponses et à leur saisie sous Excel 5.0 pour chacun des trois questionnaires. Les réponses aux deux questionnaires sur les stratégies ont été codées de façon à pouvoir les comparer (calcul de la moyenne d'utilisation de chaque type de stratégie pour chaque participant sur chaque questionnaire). Par ailleurs, les résultats de chaque participant aux exercices ont été transformés en \% de compréhension.

Les réponses aux questionnaires nous ont donné une masse de données qui ne sont pour le moment que partiellement exploitées. Par ailleurs, un compte rendu détaillé des résultats déjà obtenus dépasserait largement le cadre de cet article.

Il est cependant utile de donner à titre d'illustration du type d'information qui peut être pertinent pour notre hypothèse de travail les données d'une des études faites à partir des résultats. Après avoir calculé les profils d'utilisation des différentes catégories de stratégies pour chaque participant et sur chacun des questionnaires Q1 et Q3, nous avons relevé tous les cas pour lesquels l'utilisation d'une catégorie de stratégies directes différait de plus de 0,2 points en valeur absolue sur l'échelle de Likert entre les deux questionnaires. Cette variation dans l'utilisation des stratégies directes pouvait être en plus ou en moins. Ceci nous a permis de calculer le pourcentage de variation dans l'utilisation des stratégies directes pour chaque catégorie et pour chaque support média.

51 La figure 4 montre ces variations groupées par support et la figure 5 montre les mêmes variations groupées par type de stratégie. 
Figure 4: Changement dans les stratégies en fonction du support (regroupement par support)

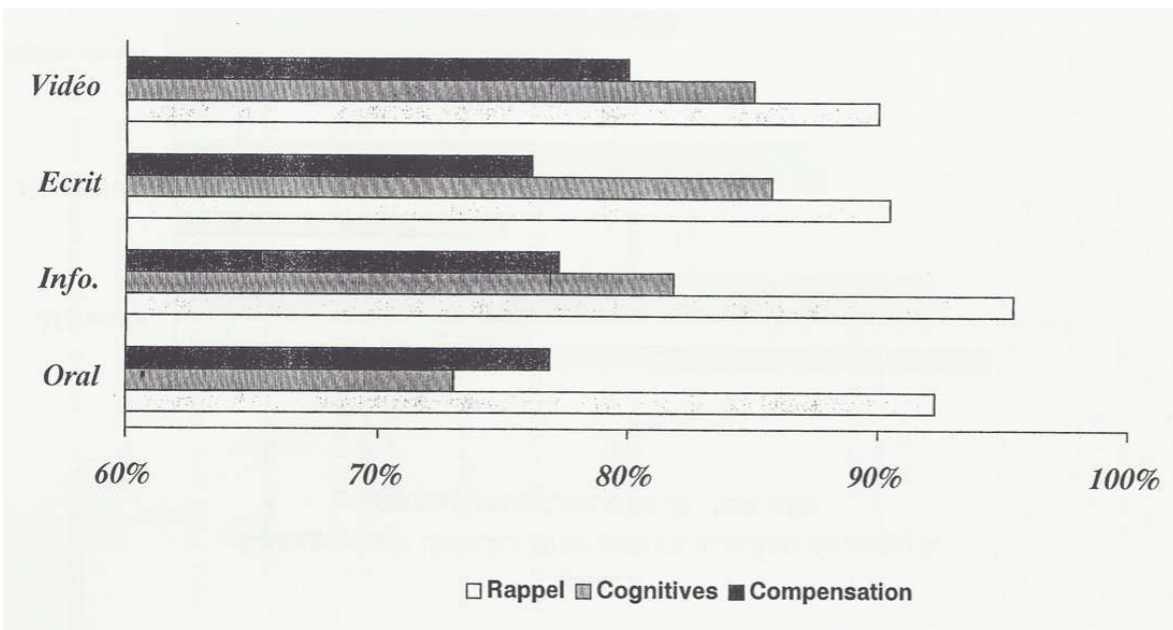

Dans la figure 4, nous pouvons constater que la variation dans l'utilisation des stratégies de compensation reste stable à travers les quatre supports ; de même que l'utilisation des stratégies de rappel. Par contre, le support cassette audio semble avoir provoqué moins de variations dans l'utilisation des stratégies cognitives que les autres supports. À tel point que pour les stratégies directes, le support oral présente un profil différent des trois autres supports.

La figure 5 montre elle aussi cette différence de variation. De plus nous pouvons voir que ce sont les stratégies de rappel qui semblent avoir été le plus modifiées pendant la tâche à accomplir, quel que soit le support média.

Figure 5: Changement dans les stratégies en fonction du support (regroupement par type de stratégie)

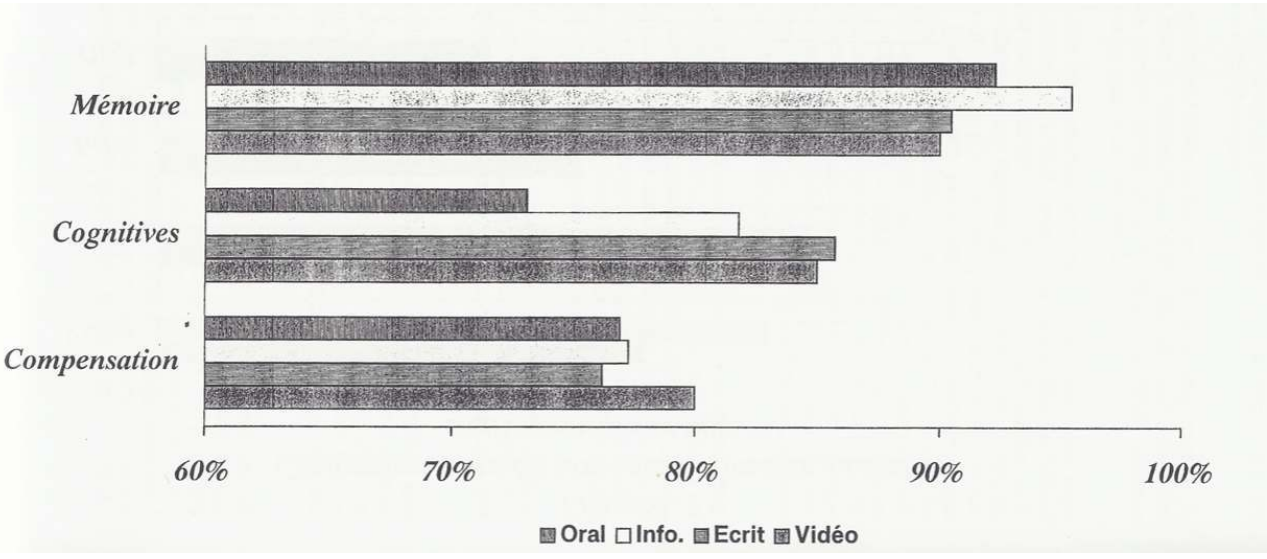

54 Ces résultats pourraient nous amener à penser que l'utilisation des stratégies cognitives reste plus stable pour les différents types d'apprenants lors d'une tâche faite en laboratoire de langues que lors d'un exercice vidéo, par exemple. En revanche, on pourrait penser que l'utilisation des stratégies de rappel varie largement, quel que soit le média.

Cependant nous ne devons pas tirer de conclusions trop hâtives car les résultats obtenus nous amènent à faire quelques remarques d'ordre méthodologique. Tout d'abord, certains items du Strategy Inventory for Language Learning se sont révélés non-pertinents. Il y a lieu 
de supprimer ces items afin d'adapter l'outil au public concerné par nos travaux. Ensuite, la corrélation entre les items des deux questionnaires s'est révélée faible. Ce qui signifie qu'il y a lieu de revoir les questions posées dans le deuxième questionnaire afin de faire valider la correspondance entre les questions et les stratégies visées.

Finalement, il faut garder à l'esprit le fait que nous avons utilisé la méthode du retour sur soi pour le premier questionnaire et celle de l'auto-observation rétrospective pour le troisième questionnaire. Il y a très probablement un décalage dans la façon dont les participants ont répondu dans les deux cas. Il nous semble donc souhaitable de revoir les items des questionnaires en fonction de cette différence. Par ailleurs, un outil pour mesurer d'autres différences individuelles des participants permettrait de contrôler des variables non mesurées ici.

En conclusion, nous avons présenté des données théoriques en rapport avec la recherche sur l'interaction entre les différences individuelles et le traitement pédagogique. Nous avons aussi abordé un certain nombre de problèmes de procédures. Cependant, il reste évident pour nous qu'un travail de plus longue haleine dans ce domaine s'impose.

\section{BIBLIOGRAPHIE}

Brown, A.L. et al. 1983. « Learning, remembering, and understanding ». In Flavill, J.H. et E.M. Markham (dir.), Carmichael's Manual of Child Psychology, Vol. 1. New York : Wiley.

Bruner, J. S. 1966. Toward a Theory of Instruction. Cambridge, MA : Harvard University Press.

Clark, R. E. 1983. « Reconsidering research on learning from media ». Review of Educational Research 53, 445-460.

Cohen, A. D. 1987. « Studying learner strategies: How we get the information ». In Wenden, A. et J. Rubin (dir.), Learner Strategies in Language Learning. Englewood Cliffs, NJ : Prentice Hall, 31-42.

Cronbach L. J. et R. E. Snow. 1977. Aptitudes and Instructional Methods. New York : Irvington Publishers, Inc.

Dulay, H.C., M.K. Burt et S.D. Krashen. 1982. Language Two. New York : Oxford University Press.

Duveau-Patureau, V. 1992. La Lettre du CLEO 47, octobre.

Galbraith, J. K. 1967. The New Industrial State. Boston : Houghton Mifflin.

Garrett, N. 1991. « Technology in the service of language learning: Trends and issues ». Modern Language Journal 75/1, 74-101.

Heinich, R., M. Molenda et J.D. Russel. 1993. Instructional Media and the New Technologies of Instruction. New York : Macmillan Publishing Company.

Kozma, R. B. 1991. « Learning with media ». Review of Educational Research 61/2, 179-211.

Naiman, N., M. Fröhlich, H.H. Stern et A. Todesco. 1978. The Good Language Learner. Ontario : Ontario Institute for Studies in Education. 
O’Malley, M. J et A. Uhl Chamot. 1990. Learning Strategies in Second Language Acquisition. Cambridge : Cambridge University Press.

Olson, D. R. 1972. « On a theory of instruction: Why different forms of instruction result in similar knowledge ». Interchange 3/1, 9-24.

Oxford, R. et D. Crookhall. 1989. « Research on language learning strategies: Methods, findings and instructional issues ». Modern Language Journal 73, 404-419.

Oxford, R. 1990. Language Learner Strategies: What Every Teacher Should Know. Englewood Cliffs, NJ : Newbury House.

Rubin, J. 1981. «Study of cognitive processes in second language learning ». Applied Linguistics 11, 118-131.

Salomon, G. 1974. « What is learned and what is taught: The interaction between media, message, task, and learner ». In Olson, D. (dir.), Media and Symbols: The Forms of Expression, Communication and Teaching. Yearbook of the National Society for the Study of Education, vol 73, part 1.Illinois : University of Chicago Press.

Salomon, G. (dir.). 1993. Distributed Cognitions. New York : Cambridge University Press.

Shannon, C. E. et W. Weaver. 1949. The Mathematical Theory of Communication. Champaign, IL : University of Illinois Press.

Skehan, P. 1989. Individual Differences in Second-Language Learning. Londres : Edward Arnold.

Snow, R. E et G. Salomon. 1968. « Aptitudes and instructional media ». Audio Visual Communication Review 16/4, 341-357.

Wenden, A. 1987. « Conceptual background and utility ». In WENDEN, A. et J. RUBIN (dir.), Learner Strategies in Language Learning. Cambridge : Prentice Hall International.

\section{Sources des exercices}

Brookes, M. et F. Lagoutte. 1989. English for Information Technology. Paris : Belin, Leçon 11 « Data Transmission ».

Upjohn, J., S. Blattes et V. Jans. 1991. Minimum Competence in Scientific English. Grenoble : Presses Universitaires de Grenoble, Unité 4 « Fish that can't see ».

Upjohn, J. 1991. Enigma 1 : Logiciel d'accompagnement de Minimum Competence in Scientific English. "The growth of salmon fishing ".

Video BBC/Omnivox. How Does a Computer Work?

\section{RÉSUMÉS}

L'article étudie l'interaction entre aptitude et traitement pédagogique dans le domaine de l'apprentissage des langues étrangères, tant du point de vue des stratégies d'apprentissage que de la technologie de formation. On décrit pour commencer le modèle du « bon apprenant », base de la recherche. Puis deux sections comparées aux différences individuelles en apprentissage des langues et aux technologies de formation fournissent un arrière-plan. Enfin, l'auteur décrit le protocole de recherche, avant d'énoncer quelques résultats.

This paper presents research carried out in order to study Aptitude Treatment Interaction as applied to foreign language learning in the area of learning strategies and instructional technology. First, the use of the Good Language Learner model as a base for the research is 
described. Then sections on individual differences in language learning and instructional technology give background information. Finally, the research protocol is described and some results are given.

INDEX

Mots-clés : méthodologie, modèle, nouvelles technologies, stratégie d'apprentissage

Keywords : learning strategy, methodology, model, new technologies

\section{AUTEUR}

\section{JANET ATLAN}

Janet Atlan enseigne l'anglais à l'IUT de Nancy 2 en Département Informatique. Elle fait des recherches sur les différences individuelles en apprentissage des langues en présence de nouvelles technologies éducatives au sein de l'équipe COSTech de l'Université de Compiègne en vue d'une thèse de doctorat. janetatlan@gmail.com 